\title{
April 2015 Pulmonary Case of the Month: Get Down
}

\author{
Michael Pham, MD \\ Karen Swanson, DO \\ Department of Pulmonary Medicine \\ Mayo Clinic Arizona \\ Scottsdale, AZ
}

\begin{abstract}
History of Present IIIness
A 59 year old woman was admitted with hypercapnic respiratory failure and an altered mental state. She had progressive "breathing issues" for the last year and was increasingly error prone with decreased mental acuity at the end of her work shift for the last 6 months. She was on oxygen at $2 \mathrm{~L}$ by nasal cannula at home and has had several admissions over the last 3 months for hypercapnic respiratory failure.
\end{abstract}

\section{Past Medical History}

Obstructive sleep apnea with continuous positive airway pressure (CPAP) intolerance, type 2 diabetes mellitus, and fibromyalgia. She is a life-long nonsmoker.

\section{Physical Examination}

Vital signs: T $36.9^{\circ} \mathrm{C}, \mathrm{P} 116$ beats/min, R 42 breaths/min, BP 134/80 mm Hg, SpO2 $93 \%$ on room air.

General: She appeared very short of breath.

Neck: No jugular venous distention.

Lungs: Clear anteriorly.

Heart: RR with a tachycardia.

Abdomen: no organomegaly or masses.

Neurologic:

- +3-to-4 of 5 strength upper and lower extremities

- Difficulty holding upright posture

- Decreased sensation in lower extremities

- $\mathrm{R}>\mathrm{L}$ lower extremity gastrocnemial fasciculations

- Hand asterixis/tremor bilaterally

- Decreased DTRs diffusely

\section{Laboratory}

ABG's: pH $7.3 / \mathrm{CO}_{2} 82 / \mathrm{pO}_{2}$ 77. Following 4 hours CPAP: pH $7.4 / \mathrm{CO}_{2} 68 / \mathrm{pO}_{2} 80$ Basic metabolic panel: $\mathrm{Na}^{+} 138 \mid \mathrm{Cl}^{-} 86$ | Creatinine 0.4

$$
\begin{aligned}
& \mathrm{K}^{+} 4.8\left|\mathrm{TCO}_{2} 44\right| \mathrm{BUN} 13 \\
& \mathrm{Ca}^{++} 4.9 / \mathrm{PO}_{4}-4.1 / \mathrm{Mg}^{++} 1.9
\end{aligned}
$$

Complete blood count: WBC 11.9 cells $/ \mathrm{mm}^{3}$, Hemoglobin $10.8 \mathrm{~g} / \mathrm{dL}$

Liver function tests, ammonia and lactate were all normal.

\section{Radiography}

Admission chest x-ray is shown in Figure 1. 


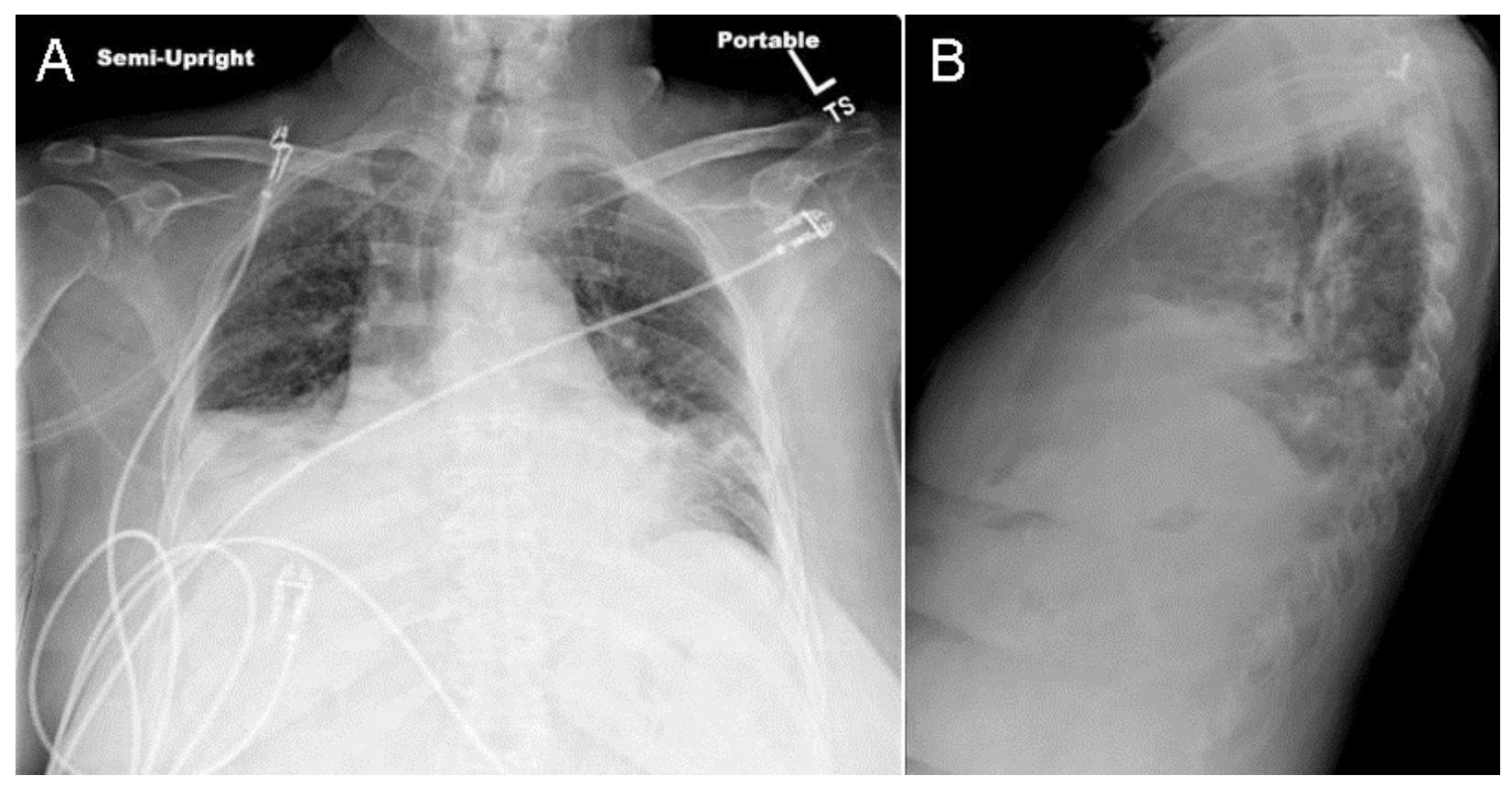

Figure 1. Admission chest x-ray.

Which of the following is/are true regarding the chest x-ray?

1. Elevated right hemidiaphragm

2. Right pleural effusion

3. Volume loss in the right hemithorax

4. 1 and 3

5. All of the above 


\section{Correct!}

\section{1 and 3}

The chest x-ray shows an elevated right hemidiaphragm. The right tracheal shift and the downward shift of the right minor fissure provide further evidence of right lung volume loss. There is no apparent pneumothorax on the left.

What would be the next appropriate step?
1. Bronchoscopy
2. Sniff test
3. Thoracic CT angiography
4. Thoracic PET scan
5. VATS 


\section{Correct!}

\section{Sniff test}

Of the tests listed, the sniff test is the most appropriate. The sniff test is a fluoroscopic exam used to assess diaphragmatic movement. Normally the diaphragm moves down during sniffing. Bronchoscopy might be reasonable if an endobronchial obstruction was suspected. Thoracic PET scan is usually done when assessing a lung mass or nodule and CT angiography is usually done when pulmonary embolism or other vascular abnormality is suspected. Video-assisted thorascopic surgery (VATS) is overly aggressive and not indicated.

The sniff test showed normal hemidiaphragm excursion on the left but paradoxical on the right (Figure 2).

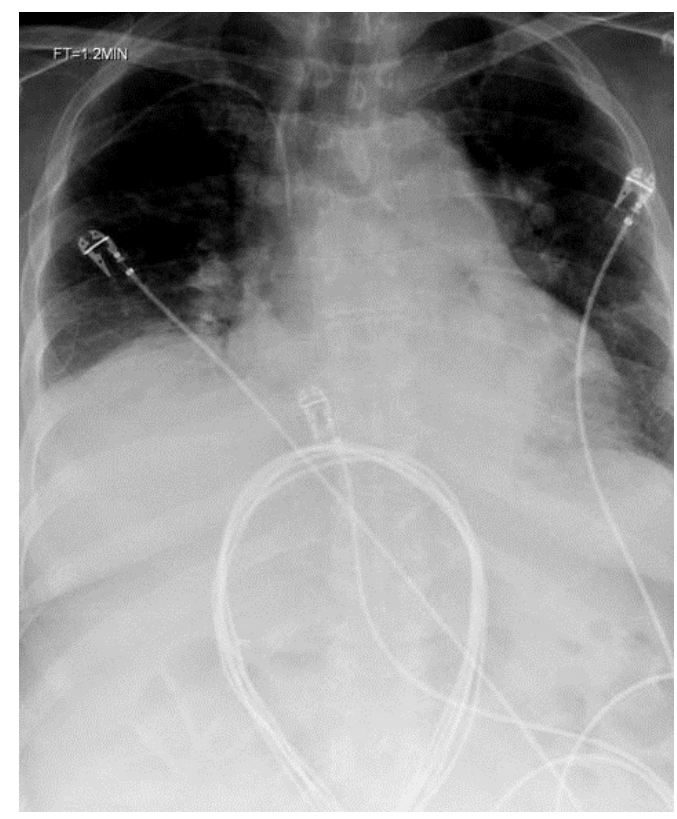

Figure 2. Static chest x-ray taking during the sniff test showing the persistently elevated right hemidiaphragm.

Neurology was consulted and suggested a lumbar puncture (Figure 3) and nerve conduction studies (Figure 4).

\section{Lumbar Puncture CSF}

\section{-Protein 64 (Normal 14-45) \\ -Nucleated Cells 3.3, (Normal 0-5) \\ - Glucose 161, normal (serum glucose 255)}

Figure 3. Pertinent findings from lumbar puncture. 


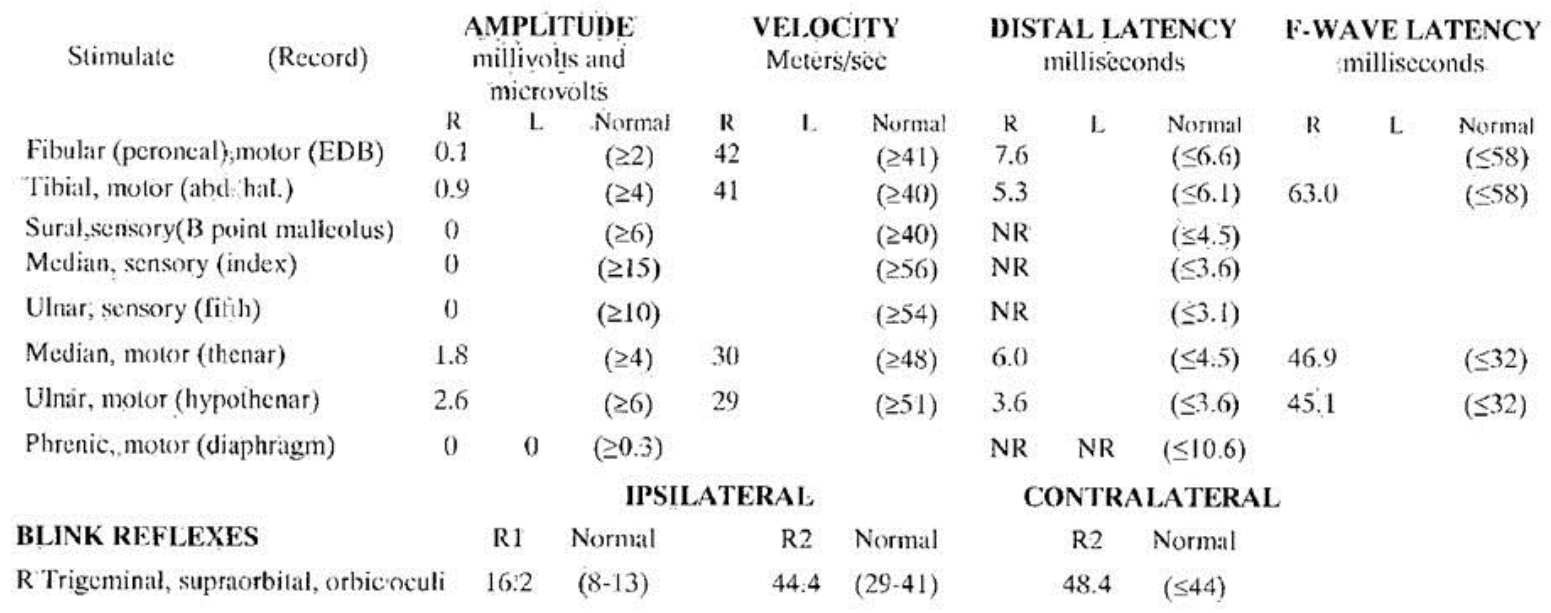

Figure 4. Nerve conduction velocity studies.

Which of the following is the best explanation of the patient's findings?

1. Chronic inflammatory demyelinating polyneuropathy

2. Dermatomyositis

3. Diabetic neuropathy

4. Duchenne muscular dystrophy

5. Myasthenia gravis 


\title{
Correct! \\ 1. Chronic inflammatory demyelinating polyneuropathy
}

The lumbar puncture shows elevated CSF protein and the nerve conduction velocity studies showed no nerve conduction to the right hemidiaphragm. Of the choices listed, the clinical and neurologic studies are most consistent with chronic inflammatory demyelinating polyneuropathy (CIDP). CIDP is an acquired immune-mediated inflammatory disorder sometimes called chronic relapsing polyneuropathy (CRP) or chronic inflammatory demyelinating polyradiculoneuropathy (1). CIDP is closely related to Guillain-Barré syndrome and acute inflammatory demyelinating polyneuropathy, and it is considered the chronic counterpart of that acute disease.

The diagnostic criteria are listed in table 1 (2).

\author{
Table 1. Diagnostic Criteria For CIDP \\ -Symmetric involvement of arms and legs \\ -Proximal with distal muscle weakness \\ -Weakness > Sensory Symptoms \\ -Diffuse reduction in DTRs \\ -CSF elevated protein without pleocytosis \\ -EMG and/or nerve Bx showing \\ demyelinating neuropathy \\ -Progressive/Relapsing course $>2$ months
}

The patient met the general criteria for CIDP. Phrenic nerve involvement has been reported with CIDP but is rare (3-5). First-line treatment for CIDP includes corticosteroids, plasmapheresis (plasma exchange), and/or intravenous immunoglobulin (IVIG) (1).

Our patient is improving slowly. She is tolerating BiPAP and is receiving IVIG and corticosteroids. She is undergoing occupational and physical therapy with plans to continue therapy at an acute rehabilitation hospital.

\section{References}

1. Dalakas MC. Advances in the diagnosis, pathogenesis and treatment of CIDP. Nat Rev Neurol. 2011;7(9):507-17. [CrossRef] [PubMed]

2. Saperstein DS, Katz JS, Amato AA, Barohn RJ. Clinical spectrum of chronic acquired demyelinating polyneuropathies. Muscle Nerve. 2001;24(3):311-24. [CrossRef] [PubMed]

3. Phrenic nerve palsy as a feature of chronic inflammatory demyelinating polyradiculoneuropathy. Stojkovic T, De Seze J, Hurtevent JF, Fourrier F, Vermersch P. Muscle Nerve. 2003;27(4):497-9. [CrossRef] [PubMed] 
4. Kawakami T, Sekijima Y, Tokuda T, Yamazaki M, Yanagisawa N. A case of chronic inflammatory demyelinating polyradiculoneuropathy complicated by phrenic nerve palsy. Rinsho Shinkeigaku. 1998;38(2):118-21. [PubMed]

5. Kimber TE, Orrell RW, King RH, Ginsberg L. Pathological findings in a patient with ventilatory failure and chronic inflammatory demyelinating polyneuropathy. $\mathrm{J}$ Peripher Nerv Syst. 2003;8(1):13-6. [CrossRef] [PubMed] 